\section{Computing I}

\section{The Internet}

MS03.01.01 THE SCIENCE, TECHNOLOGY AND ECONOMICS OF ELECTRONIC PUBLISHING IN CRYSTALLOGRAPHY. Edward N Maslen, Crystallography Centre, University of Western Australia, Nedlands 6907, Australia

Crystallographic information can be transmitted electronically at spectacular rates, allowing brief synopses to transform into detailed documents at the click of a mouse. Irksome restrictions on diagrams and tables could be eliminated. Automatic checking of data helps editors and referees to reduce errors, while those that escape detection could be corrected a posteriori. New information standards such as SGML, the Standard Graphics Markup Language, are designed to meet the evolving requirements of electronic publications. Users familiar with SGML could customise published information to suit their own needs. The possible benefits from new technology in crystallographic publishing are many and varied.

New technology can also enhance a publisher's ability to earn a living by providing improved service, but success will require the capacity to foresee the effects of change. Wrong guesses will mean investing in outmoded technology. The standard rules for good management in uncertain times apply. It is unwise to cut options unnecessarily. Because each stage in publishing has its overheads, stages should be as few as possible. Undertake full time tasks that require limited capital in-house. Sub-contract tasks that require expensive facilities on a part time basis. Assess demand, cost, and the rate at which users adapt before changing technology.

Is refereeing for electronic publishing of scientific information necessary, or should authors simply make their work accessible for comment on the network? The answer may well differ for different types of crystallographic publication. Un-refereed crystallographic data lacks the credibility derived from rigorous scrutiny. Publishers must determine which needs they are able to accommodate, and how best to meet those needs. They must disseminate information on networks that are under strain as electronic publishing increases. The capacity of critical nodes, such as the intercontinental optical fibre links, is increasing, but will it outpace the growth in demand? What protocols and technologies are likely to dominate can be assessed only for times that are short by publishing standards. Electronic publishers will survive or die in exciting times.

\section{MS03.01.02 THE ROLE OF THE WORLD-WIDE WEB IN} COMPUTATIONAL AND PHARMLCEUTICAL CHEMISTRY. George D. Purvis III, Oxford Molecular Group, Inc., Beaverton, Oregon, USA

On February 9, a Lycos search of 19 million World-Wide Web URLs found 29,053 documents containing the word chemistry. These documents allow users to study complete chemistry courses, to examine product literature, to demo the World Drug Index, to order chemicals, to list hazardous substances, to compute 3D coordinates from a 2D description (SMILES), to communicate with professional societies, to retreive supplemental material, to locate chemical patents, and to browse online journals.

University chemistry departments are providing faculty biographies, course descriptions and schedules.

Likewise, many chemical and pharmaceutical companies are using private internal web pages to distribute organizational charts, phonebooks, internal reports and research results.

Overnight, the Web has become a source of chemical news, a library of textbooks and lectures, a library catalog and more with the potential to revolutionize the practice of chemistry. Has it? Will it? Are there other or better roles for the Web? The Chemistry Information Exchange Working Group has been meeting to develop expanded roles for Web-based chemistry that will allow for the threading of applications to create active research software driven through a Web browser. This talk will review the current roles and report on the future possiblities for the Web in chemical and pharmaceutical research.

MS03.01.03 INTERNET RESOURCES FOR CRYSTALLOGRAPHY. Y. Epelboin' 1 and H.D. Flack2, 1Laboratoire de Minéralogie-Cristallographie, URA 009 CNRS, Université de Pierre et Marie Curie, Paris, France, 2Laboratoire de Cristallographie, University of Geneva, Switzerland.

The presentation will review resources available over the Internet currently and potentially useful to crystallographers. As the Internet is a very open system connecting together computers of many different types and operating systems, it is of particular importance to pay attention to the interoperability and platformindependence of potential resources.

The difficulty of assessing the quality of information will be discussed and also possible means of extracting only that which is useful. International cooperation is developing among crystallographers to solve this crucial problem.

Important Internet resources are given in the following list and those not treated elsewhere in the microsymposium or in the Congress will be discussed during the presentation.

- Remote terminal sessions (telnet)

- File transfer (ftp)

- E-mail and MIME extensions

- List servers

- Usenet newsgroups

- WWW - The World Wide Web

- Indexing and cataloguing

- Distributed (X500) directories

- Electronic Publishing

- MOO/WOO and 3D chat rooms

- Telephony and teleconferencing

- Java and VRML

Detailed information with on-line tutorials concerning the installation and use of Internet resources will be presented in the Surfing the Crystallographic Net Workshop to take place on Tuesday 13 th August during the XVII IUCr Congress.

MS03.01.04 JAVAAND ITS APPLICATIONS TO CRYSTALLOGRAPHY. John C. Huffman and John N. Huffman, Indiana University Molecular Structure Center, Chemistry Building, Indiana University, Bloomington, IN., 47405-4001

The Java programming language offers an ideal graphical interface for viewing crystallographic data on the World Wide Web. During the past year the Indiana University Molecular Structure Center (IUMSC) has installed a web based data server to allow convenient access to locally obtained crystallographic data files. In addition to having coordinate and other numeric data available in downloadable files, Java applets being developed allow users to view the molecular structures as stickfigures, ball and stick, and space-filling representations. Three dimensional views are available as stereographic pairs or red/green anaglyphs, and most modern workstations are powerful enough to allow smooth real-time rotation of the figures. Other software being developed allows the user to request publication quality figures from the server in several formats. After orienting and previewing using the Java applet, the server can be requested to generate an inage file suitable for downloading. The use of Java applets allows the server to become not only a convenient tool to store crystallographic data, but a significant aid in the interpretation and presentation of the results. The server is currently available at url: http:liwww.iumsc.indiana.edul. 